\title{
NIVELES DE INTEGRACIÓN SOCIOCULTURAL EN UN ÁREA NATURAL PROTEGIDA DE MÉXICO
}

\section{LEVELS OF SOCIOCULTURAL INTEGRATION IN A PROTECTED NATURAL AREA IN MEXICO}

\author{
Xochitl Salomé Castañeda* \\ Juan Jesús Velasco Orozco** \\ Mónica Elizama Ruiz-Torres ${ }^{* * *}$
}

RESUMEN

El objetivo de la presente investigación es analizar, desde el enfoque de la antropología social, los niveles de integración sociocultural existentes y su influencia en el manejo de recursos naturales en un Área Natural Protegida en México. Para lograrlo se empleó el método etnográfico aplicado en una comunidad campesina. Se detectaron cinco niveles de integración sociocultural que permitieron comprender las relaciones y vinculaciones entre los entornos ambientales $y$ socioculturales ${ }^{1}$.

PALABRAS CLAVE: MÉXICO * COMUNIDAD * CLASE CAMPESINA * INTEGRACIÓN CULTURAL *
INTEGRACIÓN SOCIAL * ANTROPOLOGÍA SOCIAL * ETNOGRAFÍA

ABSTRACT

The objective of this research is to analyze, from the approach of social anthropology, the levels of sociocultural integration existing and its influence on the management of natural resources in a Protected Natural Area in Mexico. To achieve this, an ethnographic method was applied in a peasant community. Five levels of sociocultural integration were

\footnotetext{
Universidad Autónoma del Estado de México, México. xoch033@hotmail.com

** Universidad Autónoma del Estado de México, México. jujevo@gmail.com

**** Universidad Autónoma del Estado de México, México. monica.ruiz.torres24@gmail.com

1 Los autores expresan su agradecimiento a los habitantes de San Mateo Almomoloa, Estado de México, por su entera disposición con el equipo de trabajo. Asimismo, se agradece a la Secretaría de Investigación y Estudios Avanzados de la Universidad Autónoma del Estado de México.
} 
detected that allowed to understand the relationships and links between environmental and sociocultural conditions.

KEYWORDS: MEXICO * COMMUNITY * PEASANTRY * ACCULTURATION * SOCIAL INTEGRATION * SOCIAL ANTHROPOLOGY $*$ ETHNOGRAPHY

\section{INTRODUCCIÓN}

Debido a la pérdida de espacios naturales a causa de actividades humanas, los debates conservacionistas han cobrado importancia $y$ se han hecho presentes dentro de las políticas públicas y los planes de desarrollo de los diferentes países del mundo.

En Latinoamérica, la creación de Áreas Naturales Protegidas (ANP) presupone un discurso conservacionista difícil de aplicar, puesto que los asentamientos humanos dentro de estas son una realidad (Ruiz, 2018). Merino y Martínez (2013) hacen notar que en México desde 1990, las comunidades locales contaban con derecho de propiedad sobre los bosques, lo que puso en el debate temas de conservación forestal. En 2001, el presupuesto federal designado a este sector se incrementó. Sin embargo, el dato de conservación es apenas un indicador parcial del éxito de la política pública, puesto que no necesariamente es sinónimo de la conservación de los ecosistemas ni de sus servicios. Adicional a esto, las implicaciones de las políticas en el plano comunitario tienen mucho que ver con las condiciones sociales de las comunidades campesinas forestales.

Entender la complejidad de las comunidades campesinas implica reconocer que las familias son la pieza clave del análisis. En este sentido, la comunidad campesina como concepto teórico proporciona una gama de posibilidades para la comprensión del entorno campesino en que viven las familias que basan su subsistencia en la obtención de recursos naturales (Castro, 2006). La presente investigación retoma el concepto de comunidad campesina pero con la condicionante de que también son comunidades forestales, es decir, dueñas de bosques, tal como lo establecen Martínez y Merino (2013). Así, el campesino se vale de la energía viva que incluye su propio trabajo y sin duda del conocimiento sobre su entorno, coincidiendo con Palerm (1998) cuando expresa que las formas campesinas de producción tienen cierta eficacia que resulta de su capacidad para usar recursos naturales, la fuerza de trabajo y los recursos menos abundantes.

Son diversos los enfoques que, desde las ciencias sociales, han analizado estrategias desarrolladas por sociedades campesinas que cohabitan dentro de ANP. Ruiz (2018), por ejemplo, a través del enfoque teórico de modos de vida, analiza una comunidad campesina dentro del Área de Protección de Flora y Fauna Nevado de Toluca. García (2012) expone la importancia que tienen las estrategias de los territorios y las políticas públicas en áreas con recursos naturales habitadas por poblaciones indígenas. Tyrtania (2009) analiza procesos adaptativos a partir de la energética social en la región zapoteca de la sierra Juárez (Oaxaca, México). Finalmente, Velasco (2002) descompone los niveles de integración sociocultural de una sociedad campesina que habita dentro de la región monarca (México), para comprender sus estrategias de adaptación.

El presente trabajo de investigación retoma el enfoque antropológico para comprender las transformaciones del tejido social en el contexto de las áreas naturales protegidas. Desde mediados del siglo xx, la ecología cultural posiciona el análisis de niveles de integración como una herramienta analítica en el estudio de cambios socioculturales dentro de cualquier sistema, es decir, categoriza en función del papel que las instituciones sociales cumplen al interior del grupo para luego "describir el orden de la vida cotidiana, para tener a su disposición el acto de significar, desde el significante en la entidad social y natural, para moldearlo y darle su valoración objetiva [...]" (Pérez, 2002, p. 152). La atribución del significado a lo material es la expresión cultural de la apropiación que 
los humanos le confieren a su entorno, el cual puede a su vez proveer de los medios necesarios para satisfacer necesidades no solo de creencia, sino de subsistencia y sobrevivencia, caso particular de los recursos naturales.

González (1997) enfatiza que la utilización del concepto de niveles de integración facilita el descubrimiento de regularidades $y$, con ello, la comparación intercultural facilita el análisis de un proceso de cambio a otro. También sostiene que, al analizar la propuesta de Steward, los niveles de integración se establecen empíricamente; en primer lugar, deben distinguirse $y$, en segundo lugar, estudiarse en sus interacciones e interrelaciones.

Boehm (2005) analizó meticulosamente la propuesta del corpus teórico de la ecología cultural y recuperó que el concepto de integración se refiere a las relaciones funcionales y sistémicas de las partes con el todo, dentro de un nivel de desarrollo, así como, la inserción de las partes en una unidad social y territorial mayor. Más tarde, Adams (2007) puntualizó que el término nivel implica una diferencia o distancia, una jerarquización social; por ello, es útil para definir las relaciones coordinadas. Propone que los niveles de integración se analicen como simplificaciones públicas; por ejemplo, la familia, el barrio, la comunidad y la nación, reflejan la relación entre el sistema social y el ambiente. Para Steward (2014), el concepto de niveles de integración es aplicable tanto a la estructura interna de las naciones como al surgimiento de nuevas clases de organización.

Con base en estos antecedentes, el propósito de la presente investigación fue analizar, desde el enfoque antropológico, los niveles de integración sociocultural existentes y su influencia en el manejo de recursos naturales en la región de la Reserva de la Biosfera de la Mariposa Monarca, México.

\section{LA REGIÓN DE LA RESERVA DE LA BIOSFERA MARIPOSA MONARCA (RBMM)}

Respecto a los eventos históricos generales de la región que hoy ocupa la RBMM, en la época novohispana, los pueblos asentados eran población indígena mazahua y otomí, tanto en el Estado de México como en el de Michoacán.
En la actualidad hay comunidades con origen indígena, así como población campesina mestiza que arribaron a la región atraídos por el trabajo en las minas, la explotación forestal y las labores agrícolas (Papousek, 1998 como se citó en Velasco, 2002). Los mazahuas dominaron la región entre los siglos VII y XII. Durante los años anteriores a la presencia española, la inestabilidad social y política dio como resultado cambios en los territorios. En los actuales municipios de Aporo, Angangueo, Zitácuaro y Maravatío, los otomíes y los mazahuas fueron desplazados por los purepechas, mientras que en Senguio habitaban mazahuas. Tlalpujahua fue ocupada por mazahuas y posteriormente por nahuas. Villa de Allende estuvo dominado por mazahuas y matlatzincas, $y$ el actual Donato Guerra por los mexicas. Una característica de la región fue el haber servido de barrera a los ataques de chichimecas en contra de los imperios mexica y purepecha (CONANP, 2001).

En el siglo xvi, la región fue dominada por el imperio español. El primero en llegar a la zona fue un soldado de apellido Parrillas. Asimismo, Cristóbal de Olid incursionó en la región y durante los siguientes años se convirtió en proveedor de madera y minerales. Así durante la colonia predominaba la actividad minera (CONANP, 2001).

En el siglo XIX, durante la lucha por la independencia, la población participó activamente. Ignacio López Rayón creó la junta de Zitácuaro y, Miguel Hidalgo y Costilla abolió la esclavitud. En 1827, Georges Ward encuentra en ruinas a Zitácuaro y expresa sus dudas acerca de su renacimiento; en 1855, los santanistas vuelven a destrozarla $y$, en 1865 , el ejército francés repite las acciones ocasionando que, posterioremente, un bando presidencial le confiera el titulo de Ciudad tres veces heroica (CONANP, 2001).

Respecto a las haciendas, en el Estado de México había dos distritos importantes: el de Ixtlahuaca y el de Valle de Bravo, el primero con 44 haciendas y 82 ranchos, y el segundo con 28 haciendas y 18 ranchos. De acuerdo con sus giros productivos, las haciendas eran "ganaderas, mineras y agrícolas, dedicándose a obrajes, explotación del bosque y el trabajo de la 
raíz de zacatón" (Velasco, 2002, p. 76). Respecto a las haciendas del Estado de Michoacán, estaban administradas por los distritos de Maravatío y Zitácuaro. En el primero, en 1889 había 34 haciendas y 464 ranchos, mientras que, en el distrito de Zitácuaro, en el mismo año había 34 haciendas y 416 ranchos. Su producción se enfocaba en la agricultura de temporal, el trabajo forestal y la ganadería a pequeña escala (Vargas, 1990, como se citó en Velasco, 2002).

En el siglo xx, "en la década de los sesenta se da en la región un gran movimiento migratorio hacia las ciudades de México y Toluca, como resultado de los procesos de industrialización de ese entonces" (Velasco, 2002, p. 79). En relación con los avances tecnológicos de la minería de la región, en 1910, había 5000 obreros en las minas. La pobreza era la condición normal de los campesinos durante la primera mitad del siglo xx (O`Dogherty, 1998 como se citó en Velasco, 2002).

En marzo de 1980 , se emite el primer decreto presidencial para la protección de los sitios de hibernación de la mariposa monarca y se declara el área como Zona de Reserva y Refugio de Fauna Silvestre. El 30 de septiembre de 1986 se emite un decreto adicional que establece un área de conservación de flora donde se precisa la superficie de 16100 ha y la zonificación actual, con lo cual se establece dos tipos de zonas protegidas: zonas núcleo donde las mariposas hibernan y zonas de amortiguamiento para proteger el hábitat forestal (SARH, 1986, como se citó en Velasco, 2002).

En 1977, se realizó la primera reunión de especialistas de la Unión Internacional para la Conservación de la Naturaleza (UICN) en EUA y se estableció como prioritaria la conservación de las áreas mexicanas de hibernación de la mariposa monarca. A partir de entonces, no es posible comprender la región sin la presencia de las Organizaciones de la Sociedad Civil (osc), tanto de orden internacional como nacional y regional, las cuales se han enfocado principalmente a la conservación del hábitat de la mariposa monarca $y$, en menor medida, al trabajo con las poblaciones de la región para aminorar las carencias sociales a través de capacitación o la implementación de proyectos económicos alternativos.

En 1992, se realizó la incorporación de la reserva al Programa de Conservación de la Biodiversidad en Áreas Naturales Protegidas Selectas de México financiado por el Fondo Global del Medio Ambiente (GEF). En 1998, por iniciativa del Gobierno de Canadá, la Red Internacional de Bosques Modelo financió la constitución y operación de la Organización Bosque Modelo Mariposa Monarca, Asociación Civil (A.c.) y se fomentaron diversos proyectos comunitarios en ejidos y comunidades indígenas para mejorar la infraestructura turística, impulsar la cría de truchas y la producción de artesanía y de alimentos orgánicos, así como, la silvicultura sustentable (Brenner, 2009).

Con el decreto de la Reserva Especial de la Biosfera del 2000, se establecieron tres zonas núcleos y dos de amortiguamiento con una extensión total de 56259 hectáreas. Finalmente, la reserva se extiende a lo largo de los límites de los Estados de Michoacán y de México. Incluye los municipios de Temascalcingo, San José del Rincón, Donato Guerra y Villa de Allende en el Estado de México; Contepec, Senguio, Angangueo, Ocampo, Zitácuaro y Aporo en el Estado de Michoacán. En total, se ubican 93 predios, de los cuales 59 son ejidos, 13 comunidades indígenas y 21 pequeñas propiedades (Galindo y Rendón, 2005). Cabe mencionar que, en el 2008, la unEsco declaró a la RBMm como Patrimonio de la Humanidad.

\section{METODOLOGÍA}

\section{ZONA DE ESTUDIO}

Para el análisis de los niveles de integración sociocultural y del manejo de recursos naturales dentro de la región RBMm se buscó seleccionar comunidades que cumplieran con los siguientes criterios:

1. Que la población estuviera ligada a las actividades relacionadas con la tierra para así considerarla como campesina (posesión, trabajo, configuraciones simbólicas). 
2. Que la población estuviera involucrada en procesos de manejo y conservación de sus recursos naturales.

3. Que la población tuviera relación constante con Organizaciones de la Sociedad Civil en los últimos dos años y con instituciones gubernamentales relacionadas con el manejo y la conservación de la ANP.

4. Que la población obtuviera beneficios económicos derivados de la conservación ambiental.

La comunidad seleccionada está situada en el municipio de Temascaltepec en el Estado de México, este último ubicado en el centro-sur de la República Mexicana, limita al norte con Querétaro, al noreste con Hidalgo, al este con Tlaxcala, al sureste con Puebla, al sur con Morelos y Ciudad de México, al suroeste con Guerrero y al oeste con Michoacán. El municipio de Temascaltepec se encuentra en el declive de las cordilleras del Xinantécatl, comúnmente conocido como Nevado de Toluca. Este territorio municipal es montañoso dado que es una prolongación del Nevado de Toluca que atraviesa al municipio de noreste a suroeste (Ayuntamiento de Temascaltepec, 2009-2012).

La zona donde se asienta la comunidad de San Mateo Almomoloa pertenece a la Subcuenca de Amanalco-Valle de Bravo que abarca una superficie de 61548,71 hectáreas e incluye al municipio de Amanalco, la mayor parte de Valle de Bravo y superficies menores de los municipios de Donato Guerra, Villa de Allende, Villa Victoria, Temascaltepec, Almoloya de Juárez y Zinacantepec. Esta cuenca tiene tanto valor ecológico, económico y social debido a que es una de las generadoras de agua más importantes para el Estado de México (CONAFOR, 2009). San Mateo Almomoloa se ubica en el Área de Protección de Recursos Naturales de las cuencas de Valle de Bravo, Malacatepec, Temascaltepec y Tilostóc. Su territorio se extiende al Parque Estatal Santuario del Agua Corral de Piedra donde se asienta una colonia de hibernación de la mariposa monarca (territorialmente no tiene predios en la zonificación de la RBMM; sin embargo, hace parte de la región).
De acuerdo con el Diario Oficial de la Federación (1941), a San Mateo Almomoloa se le dotó de tierra bajo posesión de comunidad agraria en 1937 con una extensión de 3270,80 ha, superficie que es compartida con las localidades de La Mesa (Subdelegación San Mateo Almomoloa, Las Mesas), La Comunidad, Santa Ana y Potrero de San José. En 1941 tuvo una nueva dotación de 805,89 ha que actualmente tienen un uso 100\% forestal. Esta nueva dotación dio a la población una doble posesión de tierra bajo los regímenes de comunidad agraria y ejido.

Dado lo anterior y bajo los criterios establecidos por INEGI (2010), la población de San Mateo Almomoloa se considera rural, tiene una población total de 1729 habitantes, $866(50,08 \%)$ hombres y $863(49,91 \%)$ mujeres, de los cuales $330(20,7 \%)$ son hablantes de la lengua náhuatl. Además, hay un total de 451 viviendas, de las cuales 370 son habitadas. El promedio de ocupantes por vivienda particulares es de 4,67 personas por vivienda (INEGI, 2010).

Respecto a la infraestructura y a los servicios básicos, San Mateo Almomoloa tiene una población de 119 personas analfabetas de 15 años y más, asimismo, en el mismo grupo de edad, un total de 106 personas sin escolaridad. Hay 614 personas sin derechohabiencia a servicios de salud (de acuerdo con INEGI se refiere al derecho de las personas a recibir servicios médicos en instituciones de salud públicas o privadas, como resultado de una prestación laboral). El lugar cuenta con una Unidad de Salud dependiente al Instituto de Salud del Estado de México con una atención promedio de 60 personas por semana. Sobre la disposición de agua entubada, en el censo de 2010 INEGI reportó 146 viviendas habitadas que no disponen de agua y 288 viviendas que no disponían de drenaje, siendo esta última una de las principales carencias, tanto en servicio como en infraestructura.

La actividad económica principal en San Mateo Almomoloa es la agrícola, desarrollada en las modalidades de temporal y riego. Los cultivos más comunes son: maíz, papa, chícharo, frijol, trigo, haba, avena forrajera, col $y$ lechuga. Cabe mencionar que la actividad 
agrícola es poco tecnificada: la implementación tecnológica más común es la instalación de sistemas de riego y uso de maquinaria como tractores. Los cultivos de temporal son de maíz en asociación con frijol; los de riego son haba, chícharo, lechuga y col.
La segunda actividad económica importante para la población es el turismo derivado de la hibernación de la mariposa monarca en el periodo de noviembre a marzo de cada año en el centro turístico Piedra Herrada propiedad del ejido.

FIGURA 1

\section{UBICACIÓN DE LA REGIÓN DE LA RBMM Y LA COMUNIDAD DE ESTUDIO}

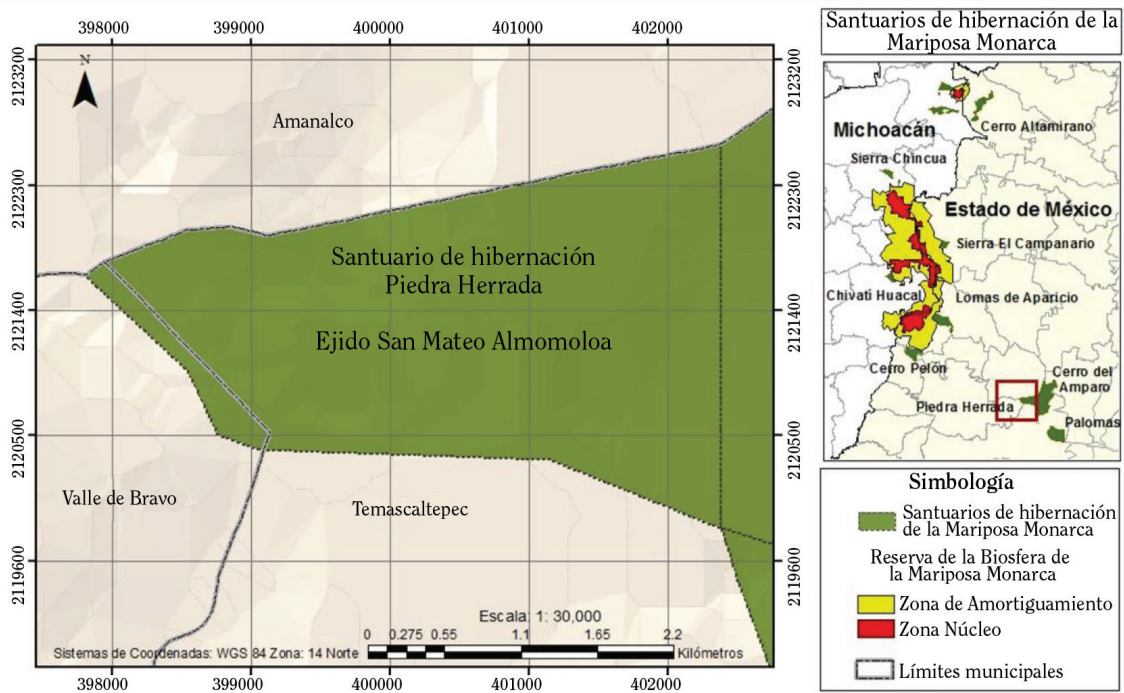

Fuente: Elaboración propia con base en los datos geográficos del Registro Agrario Nacional (2019).

\section{MÉTODO ETNOGRÁFICO}

Los datos contenidos en la investigación fueron obtenidos a través del método etnográfico que nace en el seno de la antropología social y el cual está orientado a comprender que las tradiciones, los roles, los valores y las normas del ambiente en que se vive se van internalizando poco a poco y generan regularidades que pueden explicar la conducta individual y grupal en forma adecuada (Martínez, 2007). Como metodología, la etnografía busca ofrecer descripción y análisis de determinados aspectos de la vida social teniendo en consideración los significados asociados por parte de los propios actores.

Restrepo (2016), considera que "lo que busca un estudio etnográfico es describir contextualmente las relaciones complejas entre prácticas y significados para unas personas concretas sobre algo en particular (sea esto un lugar, un ritual, una actividad económica, una institución, una red social, o un programa gubernamental)" (p.16). En esta investigación, el método etnográfico cobró relevancia para identificar las relaciones, las prácticas y aquellos procesos sociales que integran la realidad sociocultural de la comunidad campesina de San Mateo Almomoloa. De esta manera, la etnografía "supone una estrategia de investigación que implica una densa comprensión contextual de un escenario para establecer conexiones y conceptualizaciones que lo vinculan con escenarios más generales" (Restrepo, 2016, p.17).

Uno de los elementos para el desarrollo del método etnográfico es el trabajo de campo. 
"El término campo se refiere al lugar en el que se observa la cotidianidad de una comunidad para recoger sobre el terreno las informaciones que, directamente, les proveen los propios individuos" (Romero y Hernández, 2015, p. 73).

El trabajo de campo es un conjunto de operaciones que estriba básicamente en producir datos sobre el terreno (Jociles, 2018). Para el caso de la presente investigación, el trabajo de campo se organizó en tres periodos en función del calendario anual de la migración de la mariposa monarca: el primero de enero a julio de 2014 , el segundo de enero a febrero de 2015 y el último durante noviembre de 2017.

\section{SELECCIÓN DE LA MUESTRA}

En la localidad de estudio se tiene una población total de 370 familias con un promedio de 4,6 integrantes cada una (INEGI, 2010). Sin embargo, el Registro Agrario Nacional (2010), en la Carpeta Básica del padrón interno de San Mateo Almomoloa, reconoce a 148 dueños de tierras (86 ejidatarios y 62 con título de comunidad agraria), lo que representa el 40\% de lo que reconoce INEGI. Debido al objetivo de esta investigación, se decidió trabajar con familias incluidas en el Registro Agrario Nacional, es decir, aquellas cuyo tipo de propiedad social de la tierra fuera ejidataria y comunal.

A través de un muestreo no probabilístico de tipo discrecional, se seleccionaron a 15 familias campesinas que representan el 10,1\% del total en el Registro Agrario Nacional, y el $4 \%$ comparado con el total de familias que habitan en la comunidad bajo estudio. Estas familias se dividieron en dos tipos: las familias tipo $1(\mathrm{~N}=10)$, que representan el $66,7 \%$ de la muestra seleccionada, han sido beneficiarias de programas detonados por osc, sus miembros han trabajado en el parador turístico y al menos uno de sus integrantes ha asistido a capacitaciones impartidas por personal de instituciones gubernamentales; las familias tipo $2(\mathrm{~N}=5)$, que representan el 33\% de la muestra seleccionada, nunca han sido beneficiadas con proyectos promocionados por osc y ninguno de sus miembros ha trabajado en el parador turístico o se ha relacionado con actividades de reforestación o mantenimiento del bosque, entre otras.
Además de las familias contenidas en la muestra, se realizaron 11 entrevistas en profundidad a informantes clave elegidos que cumplieran con al menos una de las siguientes características: autoridad agraria, representante de osc, líder social, que ostentara cargos directivos en el parador turístico o en la empresa forestal de la comunidad.

\section{DISEÑO DEL CUESTIONARIO/GUÍA DE OBSERVACIÓN}

Se diseñaron dos formatos de entrevistas en profundidad. El primero, dirigido a las familias, tuvo un total de 12 preguntas abiertas divididas en 3 secciones: i) características socio económicas de la familia (se preguntó sobre la edad de los integrantes de la familia, la ocupación, el nivel educativo, las fuentes de ingresos haciendo énfasis en las actividades agrícolas $y$ forestales); ii) actividades de las osc en la comunidad (se preguntó sobre el conocimiento que tienen del trabajo que realizan las osc dentro de la comunidad, si en los últimos cinco años algún miembro adulto de la familia se vio beneficiado con algún proyecto o programa impulsado por osc, los cambios que se perciben en la organización de la comunidad cuando no había presencia de osc $y$, ahora que sí las hay, sobre las acciones específicas que el informante conoce de las osc y su temporalidad); $y$ iii) uso y manejo de los recursos naturales (se preguntó si el entrevistado considera que la hibernación de la mariposa monarca beneficia a la comunidad $y$, en particular, a su familia, se consultó sobre las actividades forestales que las familias realizan durante un año, sobre los impedimentos $y$ la permisibilidad de acciones relacionadas con la recolección de material vegetativo, obtención de madera, venta de tierra $y$ otras actividades en zonas forestales). Cabe decir que, adicional a este formato de entrevistas, se elaboró una genealogía para identificar la composición familiar y los tipos de propiedad de tierra de la familia.

El segundo formato de entrevista se dirigió a los informantes clave. Este tuvo un total de 11 preguntas cerradas y 3 abiertas con el objetivo de identificar cómo se desarrollan los programas y los proyectos de las osc a nivel 
comunitario y las características de la organización interna de la comunidad campesina respecto a la toma de decisiones, uso y manejo de los recursos naturales, así como, las estrategias de distribución de las ganancias de las empresas de la comunidad (el parador turístico y la empresa forestal). Se consideró que los informantes clave son las autoridades agrarias de la comunidad, los líderes de las osc y el comité del centro turístico.

Se elaboró también una guía de observación con el objetivo de detallar los contextos de desenvolvimiento de la población de manera contextual (trabajo en la empresa turística durante hibernación de la mariposa monarca, la milpa como espacio de socialización de la familia, trabajos de reforestación de la zona forestal, actividades de mantenimiento y conservación de los recursos, la asamblea como forma de organización y de toma de decisiones).

En esta investigación, todas las referencias de comunicación personal que se realizaron en el trabajo de campo se referenciarán por común acuerdo con las dos primeras iniciales que corresponden a nombre $y$ apellido paterno, seguido del año y, en su caso, los cargos que ostentaban al momento de la entrevista.

\section{RESULTADOS Y DISCUSIÓN}

En la RBMm se identificaron 5 niveles de integración de orden público definidos a partir de la interacción de la organización interna de las comunidades con instituciones externas $y$ las políticas públicas de corte ambiental bajo la figura de ANP: internacional, nacional, regional, comunitario campesino y familiar. Para Adams (2007), el término nivel implica una diferencia o distancia, una jerarquización social. Por ello, la categoría nivel es útil para definir las relaciones coordinadas. Propone que los niveles de integración se analicen como simplificaciones públicas; por ejemplo, la familia, el barrio, la comunidad y la nación reflejan la relación entre el sistema social y el ambiente.

\section{NIVEL I: INTERNACIONAL}

La riqueza natural de la región de la RBMM, compuesta por sus bosques de oyamel, pino $y$ encino, es reconocida por la UNESCO como un bien del Patrimonio Mundial Natural sobre todo por la singular relevancia faunística que posee 184 especies de vertebrados registradas (semarnat, 2019). Cabe decir que, desde el 2008, la RBMm está incluida en el Programa del Hombre y la Biosfera de la UnEsco, lo cual fue el resultado de la Convención del Patrimonio Mundial de la Humanidad y de la Convención Ramsar de Humedales, de las cuales México formó parte. A partir de entonces, el 79,6\% de las superficies de las ANP mexicanas del ámbito federal fueron reconocidas por al menos una institución internacional (Bezaury-Creel y Gutiérrez, 2009).

\section{NIVEL II: NACIONAL}

Este segundo nivel tiene que ver con la administración de los recursos financieros y con la política pública de conservación. En México, la Secretaria del Medio Ambiente y Recursos Naturales (SEMARNAT) establece que las ANP deben contar con un Programa de Manejo (PM) y otorgar su adminisTRAción a otras instancias (gubernamentales o no gubernamentales), previa opinión del Consejo Nacional de Áreas Naturales Protegidas (CNANP) (Riemann, Santes y Pombo, 2011).

Estos programas de manejo deberían poner especial énfasis en que la política de establecimiento (asentamiento humano) de las ANP considere a la población residente como un componente fundamental (Riemann, Santes, y Pombo, 2011). Sin embargo, investigaciones como la de García (2012), demuestran que en los bosques de la sierra Juárez de Oaxaca, la SEMARNAT "se ha extendido notablemente en niveles que le permiten tener mayor control de los recursos agua, bosque y suelos fundamentalmente. Todo ello se traduce en menor margen de acción de las comunidades campesinas respecto al control de esos recursos" (p. 109).

En la comunidad de estudio, la percepción no dista de lo expresado por García (2012), tal y como lo menciona un informante: "los que más nos han dado proyectos son los de SEMARNAT año con año dan cursos a los guías, dan cursos de primeros auxilios, van a poner un vivero $y$ nos dicen que tenemos que hacer 
brechas y reforestaciones" (Informante A.G., comunicación personal, 24/01/2015).

Así, los resultados de la presente investigación identifican que a través de la SEMARNAT se controlan las acciones de aprovechamiento $y$ de explotación de los campesinos que viven en ANP. Además, esas acciones se norman en el Programa de Manejo de la Reserva de la Biosfera Mariposa Monarca (2001), por lo que los usos $y$ las costumbres de las comunidades indígenas respecto al control de los recursos naturales se amalgaman con lo establecido en la normatividad federal.

\section{NIVEL III: REGIONAL}

En el tercer nivel de integración, se sitúa el trabajo de las osc en las ANP que representan importantes fuentes de apoyo adicional
(Bezaury-Creel y Gutiérrez, 2009). Estas instituciones junto con las académicas, aportan gran parte de los estudios y de las investigaciones realizados en las ANP y se responsabilizan directamente del desarrollo de una parte de los proyectos que se efectúan con las comunidades que residen en las áreas y su periferia, lo que representa un valor agregado sumamente importante en la operación de las mismas.

En la región de la RBMM, se observó que el papel de las osc está ligado de manera interna a las comunidades campesinas y que estas son el puente de comunicación entre el resto de las instituciones gubernamentales. Por lo tanto, su impacto es transversal porque incide en todos los niveles identificados. La tabla 1 muestra las 37 osc identificadas en la región de RBMM cuyo objetivo es intervenir en las actividades de manejo ambiental.

TABLA 1

ORGANIZACIONES DE LA SOCIEDAD CIVIL QUE TRABAJAN

EN LA REGIÓN DE LA RESERVA DE LA BIOSFERA MARIPOSA MONARCA

NOMBRE DE LA ORGANIZACIÓN DE LA SOCIEDAD CIVIL

1. Alianza de Ejidos y Comunidades RBMm A.c.

2. Monarca A.c.

3. Nukello Langini A.c.

4. Espacios Naturales y Desarrollo Sustentable A.c.

5. Organización Cultural Internacional A.c.

6. Servicios Alternativos para la Educación y el Desarrollo A.c.

7. Viva México A.c.

8. Espacio Autónoma A.c.

9. World Wide Fund for Nature (Programa México).

10.Asociación Nacional para el Desarrollo Integral, Sustentable y Alternativo. Trabajo Científico Aplicado A.c.

11. Fundación Nacional para la Conservación del Hábitat Boscoso de la Mariposa Monarca A.c.

12. Bosque Modelo Mariposa Monarca A.c.

13. ECOTECNIS A.C.

14. Vasco de Quiroga presencia en el siglo XXI A.c.

15. Consultores y Constructores (COSECI S.c.).

16. PRONATURA A.C.

17. ALTERNARE A.C.

18. Gestión Ambiental y Programa para el Desarrollo sustentable (Gapdes s.c.).
19. Patronato Pro-Valle de Bravo A.c.

20. Fondo Pro-cuenca Valle de Bravo A.c.

21. BIOMA A.C.

22. Patronato Pro-Tradiciones Vallesalias A.c.

23. Asociación Mexicana de Profesionales Forestales A.C.

24. Red Internacional de Metodologías de Investigación de sistemas de Producción (RIMISP A.c.).

25. Voluntariado Internacional Vive México A.c.

26. Monarca Butterfly Santuary Fundation.

27. Fundación Telmex.

28. Biocenosis A.c. Región monarca.

29. Unidad de Manejo Forestal cuenca Lerma A.c.

30. Proyectos Itinerantes s.c.

31. Red Mexicana de Organizaciones Campesinas Forestales A.C.

32. Reverdece A.C.

33. Fungus A.C.

34. Fundación ACIR.

35. The Monarcha Effect.

36. Ingenieros Agrónomos para el desarrollo rural A.C.

37. Más que Monarcas.

Fuente: $\quad$ Elaboración propia con base en los registros de participantes Foros Monarcas (2005, 2006, 2007, 2008); Velasco (2002) y datos de trabajo en campo 2014-2016. 
Se identificaron 6 osc en la comunidad campesina de San Mateo Almomoloa (nombres subrayados de la tabla 1 ), lo que representa el $16,2 \%$ del total de osc identificadas en la región de la RBMm. Todas estas han detonado proyectos o programas con la población focalizados principalmente en el centro turístico $y$ en el manejo de los recursos forestales en el periodo de 2005 a 2016.

La FUNACOM trabajaba aquí por allá del 2005 y 2008 recuerdo que fue de las primeras, en aquel tiempo ayudó con las reforestaciones masivas en la zona de del parador turístico, trajo a muchachos de otros países, se quedaban a dormir en el bosque, trató de conseguir dinero de empresas privadas, con gente que trajo intentó ampliar la oferta turística con el rescate de lengua y temazcal (Presidente del comisariado ejidal, comunicación personal, 10/07/2014).

La osc más influyente en la región de la RBMm es WWF (Programa México), la cual:

...se ha encargado de organizar los foros regionales para incluir la participación tanto de académicos como ejidatarios, comuneros, instituciones de gobierno y público en general y que además; se dedica a la gestión del Fondo para la Conservación de la Mariposa Monarca, apoyado por donaciones de la Fundación Packard, la SEMARnat, así como por los gobiernos de los estados de Michoacán y de México (Brenner, 2009, p. 278).

Adicional, a nivel comunitario (nivel IV), esta organización ha tenido una participación activa en los últimos años (a partir del 2014). Otras han tenido episodios de incidencia más cortos y con resultados menos concretos. Al referir a COSECI un informante refirió:

De esa organización ya no quiero ni hablar, ya nos enteramos de cuánto dinero les dieron para el parador turístico y la verdad lo que hicieron no lo vale, hasta queríamos demandarlos, pero eso sí cuando a mí me pregunten diré que no los recomendamos y aquí ya no entran (Presidente del comisariado ejidal, comunicación personal, 10/07/2013).

A diferencia de los hallazgos hechos por Tyrtania (2009) y García (2012), en la RBMM las OSC juegan un papel fundamental en el intercambio de información entre el exterior y el interior.

\section{NIVEL IV: COMUNIDAD CAMPESINA}

En el cuarto nivel se identificaron las estrategias campesinas de integración que vinculan a la comunidad campesina con el exterior y que le permiten su subsistencia.

En el nivel de comunidades campesinas existe un sistema de gobernanza local cimentado en la asamblea (ejidatarios, comuneros y asambleas públicas) para la regulación del uso $y$ del manejo de los recursos naturales. La toma de decisiones se basa en los usos y en las costumbres de los pueblos indígenas, relacionados con las manifestaciones populares de la cultura como las fiestas patronales y el sistema de cargos religiosos. Adicional, la asamblea también determina el control de las empresas comunitarias (parador turístico "Piedra Herrada" y empresa forestal) sobre los recursos. Cabe decir que en la asamblea solo participan las personas con derechos agrarios: los hijos y las esposas no tienen voto como ocurre en otros ejidos y comunidades (García, 2012).

Este nivel de gobernanza coincide con lo analizado por García (2012), quien también identificó que la asamblea, al respaldarse en la Ley Agraria, determina el manejo forestal y se basa en estructuras comunitarias para la toma de decisiones relacionadas con el territorio. Es un espacio político en el que se conjunta el poder y el control. "Ya se van a ir las mariposas $y$ ahorita toda la gente anda de malas, ya quieren que se reparta el dinero en la Asamblea, pero la verdad son puros pleitos, todos quieren ganancia y no salen las cuentas" (encargado del centro de interpretación de la monarca, comunicación personal, 8/02/2015).

Este nivel comunitario participa activamente en actividades turísticas de conservación. Sin embargo, no debe perderse de vista que 
estas son alternativas que se impulsan por el gobierno de los tres órdenes para evitar que las comunidades exploten los recursos naturales. Las familias están integradas en la agenda local de conservación ambiental, aunque su conocimiento sobre la normatividad ambiental (enmarcados en el primer $y$ en el segundo nivel) es limitado. El 73\% de las familias manifestaron que, al menos una vez en el último periodo de hibernación, han empleado a uno de sus miembros mayores de edad en el centro turístico.

La organización y el funcionamiento del centro turístico recaen en un comité que se elige en la asamblea de ejidatarios. Dicho comité se encarga de emplear a los encargados de la zona de estacionamiento, taquilla, restaurante, tienda artesanías, centro de interpretación de la mariposa monarca, quiosco, venta de comida al aire libre, guías y caballerangos. Para elegir a los empleados, cada ejidatario (86) tiene la posibilidad de emplearse o contratar a alguien que trabaje a su nombre. Al final de la temporada de hibernación (de enero a marzo), se paga a quienes se emplearon en las actividades señaladas $y$ el resto de las ganancias se reparten en partes iguales entre todos ejidatarios; por ejemplo, en el 2015 cada ejidatario recibió un total de 10 000 pesos al final de la temporada.

El centro turístico emplea aproximadamente 250 personas a las que se suman otras 15 que improvisan espacios para la venta de comida fuera del centro. Algunas familias ven en las acciones de conservación y en el turismo la vía para la mejora de su condición de vida:

Mi hijo estudia en la universidad para administrador de empresas, lo mandé a estudiar porque tengo un terreno enfrente del parador turístico y me gustaría que tuviéramos un negocio más grande, aquí falta mucho el trabajo, si no trabajas en las mariposas, no puedes hacer nada más. El campo no rinde, solo el de riego (informante M.s., comunidad personal, $22 / 05 / 2014)$.

La empresa forestal que funciona a través del programa de manejo forestal autorizado $y$ regulado por SEMARNAT y CONAFOR, respectivamente (permiso a partir del 2012, con duración de diez años), incluye entre sus actividades programas de reforestaciones anuales y obras de conservación de suelo; sin embargo, el 100\% de las familias tipo 2 (5 familias que represen$\tan$ el 33,3\%) refirieron no haber participado por ser exclusivas de ejidatarios y comuneros. Otras actividades realizadas son las de mantenimiento: reacomodos de material vegetativo, podas en veredas, monitoreo de ramoneo y brechas contra incendios. Al respecto, el $80 \%$ de las familias entrevistadas refirieron haber participado en el último año y haber obtenido remuneración económica por sus jornales.

Los trabajos en el bosque son entre abril $y$ diciembre de cada año, nosotros como ejido tenemos un permiso de aprovechamiento forestal, ahí se emplean algunas personas de la comunidad y se les paga, también reforestamos cada año y se paga por cada árbol plantado, hacemos brechas, acomodos, líneas negras y todo lo que nos dicen nuestros técnicos. Hay gente de aquí que ya se ha capacitado en otros estados $y$ pretendemos ser una empresa en el futuro con nuestro aserradero (Presidente del comisariado ejidal, comunicación personal, 10/07/2014).

A diferencia de las comunidades analizadas por García (2012), donde los recursos obtenidos de los programas de manejo se destinan a beneficios comunes como fiestas patronales, escuelas y otros servicios, en las comunidades campesinas dentro de la RBMm se destinan únicamente al pago de jornales. Es importante señalar que el objetivo de dichos programas es la protección de la cuenca hidrográfica, la conservación de la biodiversidad, los beneficios ambientales para toda la población y los beneficios económicos de la venta de madera únicamente para los ejidatarios. Las autoridades agrarias refirieron que, una vez que se vende la madera en pie, las ganancias se reparten en partes iguales entre los ejidatarios, así como, las ganancias del centro turístico. Los beneficios de las empresas de la comunidad se amplían a diferentes miembros de la familia debido a que los propietarios de tierra ejidal pueden subcontratar a sus parientes: 
Mi abuela es muy grande para trabajar, por eso yo estoy en su lugar. Por mi trabajo en el restaurante me paga $\$ 120.00$ al día, me dedico a preparar comida. A ella por semana le van a repartir sus ganancias por estar en el restaurante de encargada $y$ al final de la temporada también va a recibir su pago como ejidataria (informante M.Z., comunicación personal, 22/05/2014).

\section{NIVEL V: FAMILIAR}

En este nivel de integración, el uso/apropiación y manejo de los recursos naturales se relaciona directamente con tipo de propiedad social de la tierra que ostenta la familia. En el gráfico 1, se observa el tipo de posesión de la tierra entre las familias de la RBMm. Cabe decir que las familias agrupadas en el tipo 1, manifestaron que obtienen mayores recursos económicos por poseer títulos de propiedad, mientras que las de tipo 2 los obtienen únicamente por la temporada de hibernación de la mariposa monarca. En el siguiente testimonio clasificado como Familia tipo 2, se visualiza la diversidad de actividades realizadas para la obtención de beneficios económicos:

En el año tengo varias actividades que me dejan dinero o comida, la más importante es el trabajo en el campo, me empleo como peona en las cosechas de los vecinos o de mis parientes, también me voy a trabajar a Cerro Colorado a los cultivos y también a veces he sido guía cuando están las mariposas (informante E.B., comunicación personal, 24/01/2015).

\section{GRÁFICO 1 \\ POSESIÓN DE LA TIERRA DE LAS FAMILIAS \\ EN UNA COMUNIDAD CAMPESINA DE LA RBMM}

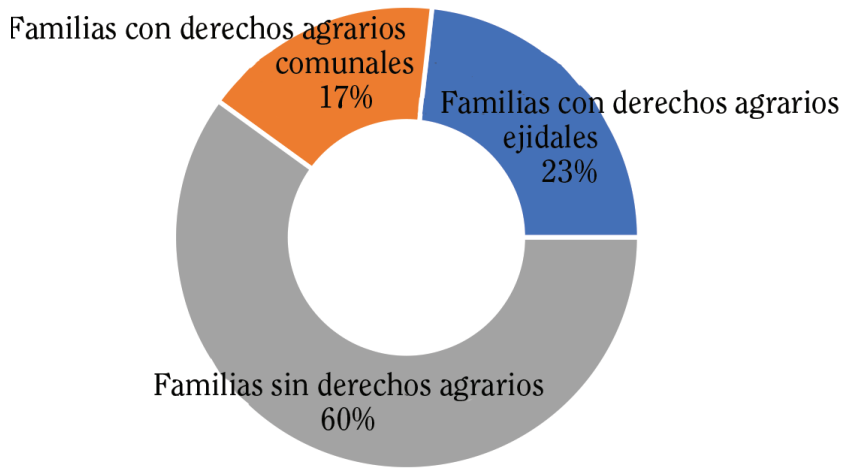

Fuente: Elaboración propia con datos de INEGi (2010), carpeta básica de ejidatarios y comuneros de San Mateo Almomoloa, última revisión en 2018.

Las familias también han desarrollado estrategias de subsistencia campesina que se insertan de manera estratégica en las formas de aprovechamiento de los recursos y que se extienden a las actividades empresariales de conservación. El gráfico 2 muestra que del total de la muestra (quince familias), el $66,7 \%$ (diez familias) manifestaron haber trabajado en reforestaciones que les han generado recursos económicos y el $66,7 \%$ dijo haber participado en algún tipo de proyecto o programa implementado por osc sin que necesariamente el beneficio fuese económico (relación con el nivel III). Las estrategias de subsistencia campesina son muy variadas y se dan debido a las historias particulares de las familias, pero pueden coincidir 
en la percepción que se tiene de los recursos naturales de la comunidad como pieza clave en su diseño de proyecto de vida:

Tiene más de 10 años que me separé de mi esposo, ese día comencé a planear lo que tengo hoy, hoy mis hijas están en la escuela, tengo mi restaurante, ya casi termino mi casa, y soy la tesorera del comisariado ejidal, la gente me respeta, antes me veían mal por estar sola por haber corrido a mi marido, pero ahora las cosas han cambiado, no todos tenemos la suerte de tener una familia de dos padres eso es lo que yo les enseño a mis hijos, a mí me gustaría que mi hija la que se fue a estudiar a Michoacán para técnico forestal algún día regrese con su papel y sea ella la que aquí nos enseñe a cómo cuidar el bosque (informantes A.R., comunicación personal, 22/05/2014).

\section{GRÁFICO 2 \\ ACCIONES DE CONSERVACIÓN/PROTECCIÓN CON REMUNERACIÓN ECONÓMICA DE LAS FAMILIAS CAMPESINAS}

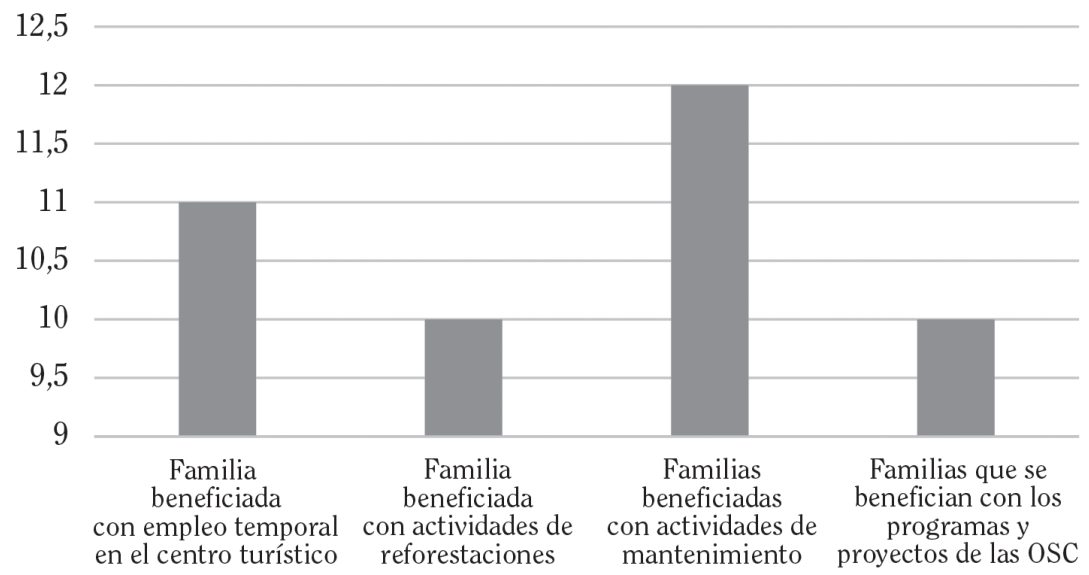

Fuente: Elaboración propia con base en el trabajo de campo, 2015.

Es importante hacer notar que todas las familias entrevistadas realizan actividades agrícolas, pero también todas realizan actividades no agrícolas, en tanto que el 66,7\% (diez familias) de las familias, además de las actividades propias de su parcela, se emplean como jornaleros en parcelas de riego que no son propias: el 100\% manifestó recolectar hongos durante los meses de junio, julio y agosto, plantas medicinales (durante todo el año) y de ornato (durante las festividades de días de muertos en noviembre), así como extracción de tierra de monte al menos una vez al año para consumo propio o para venta.

En mi familia nos dedicamos a sacar papas [cosechar papas], en los pueblos donde hay riego aquí no hay, solo maíz y chícharo de temporal, tenemos nuestro terrenito y nos dedicamos a eso; a la siembra y cosecha, también yo me he dedicado a las mudanzas, le ayudo a un señor de Toluca pero ese no es un trabajo fijo solo de vez en cuando (informante E.Z., comunicación personal, 22/05/2014).

Respecto a las remesas, el $60 \%$ de las familias (nueve) dijo recibir dinero entre una $y$ dos veces cada seis meses por familiares que viven fuera de la entidad o fuera del país (gráfico 3). 


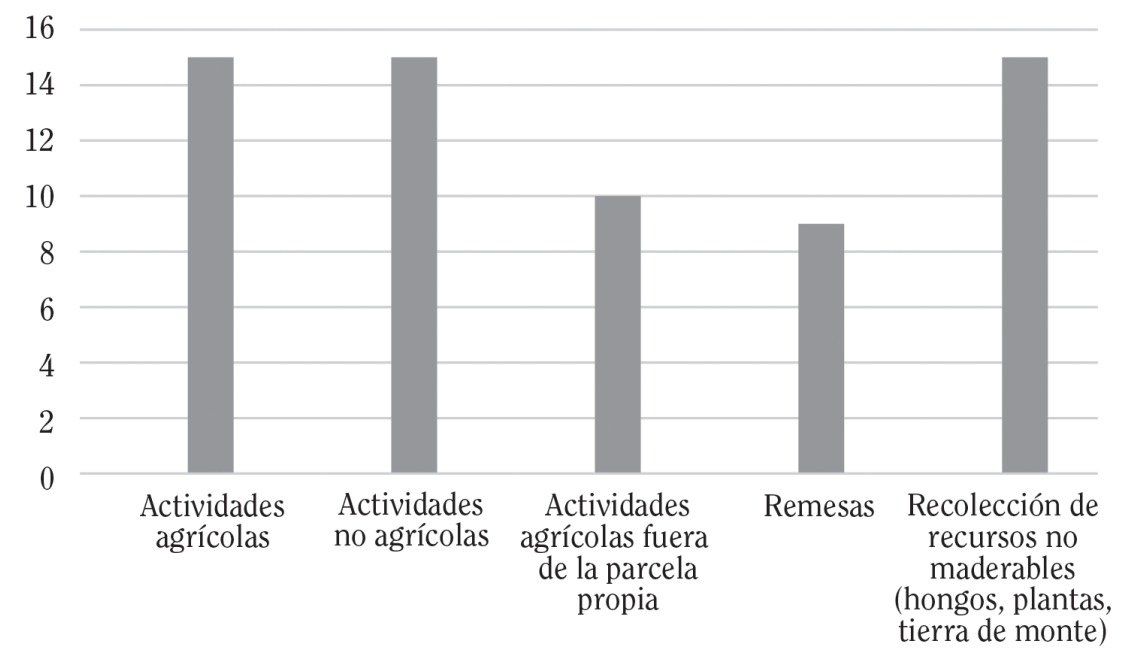

Fuente: $\quad$ Elaboración propia con base en el trabajo de campo, 2015-2016.

Lo anterior refleja que las actividades de las familias campesinas como medios de subsistencia son el resultado de la experiencia: "este sistema de conocimiento refleja la experiencia de muchas generaciones, así como los mecanismos de resolver problemas por parte de los agricultores en todo el mundo" (García y Padrón, 2012, p.67).

\section{CONCLUSIONES}

Durante la investigación se detectaron cinco niveles de integración sociocultural ligados estrechamente. En el nivel I se identificó la interrelación entre la comunidad campesina y las instituciones internacionales que fomentan la conservación del hábitat de la mariposa monarca.

En el nivel II, se identificaron las instituciones mexicanas y toda su normatividad que fomentan y permiten el aprovechamiento de los recursos naturales a la población.

En el nivel III se identificó la relación entre las osc y las comunidades campesinas, la cual se catalogó como estrecha, ya que más de la mitad de las familias entrevistadas manifestaron haber sido partícipes de los procesos o actividades que implementan dichas instituciones.
En el nivel IV se identificó a la comunidad campesina, dentro de la cual se desarrolla la gobernanza interna de la población mediante lo dispuesto en la Ley Agraria, pero también en los usos y en las costumbres de las comunidades indígenas.

El nivel $v$ corresponde a las familias $y$ a sus actividades que las vinculan con el resto de los niveles (por ejemplo de servicios turísticos) $y$ aquellos relacionados con la conservación/ protección de los recursos forestales, lo que indica una fuerte relación entre las familias y el trabajo en la zona forestal basada en los beneficios monetarios.

\section{REFERENCIAS}

Adams, R. N. (2007). La red de la expansión humana. Centro de Investigaciones y Estudios Superiores en Antropología Social, Universidad Autónoma Metropolitana, Universidad Iberoamericana.

Ayuntamiento de Temascaltepec. (2009-2012). Plan Municipal de Desarrollo Urbano de Temascaltepec. https://www.ipomex. org.mx/recursos/ipo/files_ipo/2011/8/9/ a9c593a62097576a94f163b9f99e3bb2.pdf 
Bezaury-Creel, J., D. y Gutiérrez, D. (2009). Áreas naturales protegidas y desarrollo social en México. Capital natural de México, vol. II: Estado de conservación y tendencias de cambio (385-431). Conabio. https://www.biodiversidad.gob. $\mathrm{mx} / \mathrm{v}_{-}$ingles/country/pdf/CapNatMex/ Vol\%20II/II09_Areas\%20naturales\%20 protegidas $\% 20$ y $\% 20$ desarrollo $\% 20$ social $\% 20$ en $\% 20$ Mex.pdf

Boehm, B. (2005). Buscando hacer ciencia social. La antropología y la ecología cultural. Relaciones, XXVI (102), 63-128.

Brenner, L. (2009). Aceptación de políticas de conservación: El caso de la biosfera mariposa monarca. Revista Economía, sociedad y territorio, 30 (IX), 259295. http://www.redalyc.org/articulo. oa? id=11111267002

Castro, F. (2006). Colapsos ambientales transiciones culturales. Universidad Nacional Autónoma de México.

Comisión Nacional Forestal-ConAFor. (2009). Evaluación Rural Participativa Ejido San Mateo Almomoloa, Municipio de Temascaltepec. Comisión Nacional Forestal y ECOTECNIS SA DE CV.

Comisión Nacional de Áreas Naturales Protegidas-conanp. (2001). Programa de Manejo Reserva de la Biosfera Mariposa Monarca, México. https://simec.conanp. gob.mx/pdf_libro_pm/40_libro_pm.pdf

Diario Oficial de la Federación. (1941). Resolución presidencial de la comunidad agraria de San mateo de los ranchos y sus anexos. Registro Agrario Nacional.

Galindo, C. y Rendón, E. (2005). Danaidas: Las maravillosas mariposas monarcas. Telcel publicación especial.

García, A. (2012). Control y poder en los bosques de la sierra Juárez de Oaxaca [Tesis para obtener el grado de maestra en antropología]. Universidad Autónoma de México, Instituto de Investigaciones Antropológicas, Distrito Federal, México.

García, L. y Padrón, J. (2012). El sistema de conocimiento popular agrícola en el contexto del desarrollo local al nivel rural. Agroalimentaria, 18(35), 61-73. https://www.redalyc.org/articulo. oa?id=1992/199224435006

González, A. (1997). La influencia de la antropología estadounidense en México: el caso de la ecología cultural. En R. Mechthild y C. Serrano (Ed), Ciencia en los márgenes (pp. 162-189). Universidad Nacional Autónoma de México.

Instituto Nacional de Estadística y GeografíaINEGI. (2010). Datos por localidad sistema ITER. http://www.inegi.org.mx/sistemas/ consulta_resultados/iter2010.aspx

Jociles, M. I. (2018). La observación participante en el estudio etnográfico de las prácticas sociales. Revista colombiana de antropología, 54 (1). http://www.scielo.org.co/pdf/rcan/v54n1/0486-6525rcan-54-01-00121.pdf

Martínez, M. (2007). Evaluación cualitativa de programas. Editorial Trillas.

Merino, L. y Martínez, A. E. (2013). El campo forestal mexicano y las comunidades forestales. En Merino Pérez, L. y Ortiz Merino, G. (Cood.), Encuentros y desencuentros las comunidades forestales y las políticas públicas en tiempos de transición (pp. 35-56). Universidad Nacional Autónoma de México, Instituto de Investigaciones Sociales, Miguel Ángel Purrúa.

Palerm, A. (1998). Antropología y marxismo. Centro de Investigaciones y Estudios Superiores en Antropología Social.

Pérez, R. (2002). Construir el espacio. En Pérez Taylor, R. (Comp.), Antropología y complejidad (pp. 139-168). Gedisa.

Registro Agrario Nacional Registro Agrario Nacional. (2010). Carpeta Básica del padrón interno de San Mateo Almomoloa, Estado de México: México. Gobierno del Estado de México.

Registro Agrario Nacional. (2019). Datos geográficos de las tierras de uso común, por Estado, Formato SHAPE. https://datos. gob.mx/busca/dataset/datos-geograficosde-las-tierras-de-uso-comun-por-estado-formato-shape

Restrepo, E. (2016). Etnografía: alcances, técnicas y éticas. Envión editores. http://www. 
ram-wan.net/restrepo/documentos/libroetnografia.pdf

Riemann, H., Santes, R.V. y Pombo, A. (2011).

El papel de las áreas naturales protegidas en el desarrollo local El caso de la península de Baja California. Revista Gestión y Politica Pública, 1 (xx), 141172. http://www.gestionypoliticapublica.cide.edu/num_anteriores/Vol. XX_No.I_lersem/04_Hugo-Riemann_y_ co-autores.pdf

Romero, M. A. y Hernández, A. R. (2015). El método etnográfico y su relación con el análisis de dominio. Revista Biblios, 61, 70-84. https://doi.org/10.5195/ biblios.2015.250

Ruiz, M.E. (2018). Modos de vida rurales en el Área de Protección de Flora y Fauna Nevado de Toluca. Revista Ciencia ergosum, 26, 1-8. https://doi.org/10.30878/ ces.v26n1a5

Secretaría de Medio Ambiente y Recursos Naturales-SEMARNAT. (2019). Reserva de la
Biosfera Mariposa Monarca. https://www. gob.mx/semarnat/articulos/reserva-dela-biosfera-mariposa-monarca-79228

Steward, J. H. (2014). Teoría del cambio cultural: la metodología de la evolución multilineal. Universidad Iberoamericana, Universidad Autónoma Metropolitana, Centro de Investigaciones y Estudios Superiores en Antropología Social.

Tyrtania, L. (2009). Evolución y sociedad. Termodinámica de la supervivencia para una sociedad a escala mundial. Universidad Autónoma Metropolitana y Juan Pablos Editores.

Velasco, J. J. (2002). Subsistencia Campesina $y$ desarrollo sustentable en la región monarca. Universidad Autónoma del Estado de México.

Fecha de ingreso: 15/11/2019 Fecha de aprobación: 07/10/2020 